\title{
Diallel Analysis of Combining Ability for Yield and Yield Contributing Traits over the Environments in Maize (Zea mays L.)
}

\author{
Avinash Kumar ${ }^{1 *}$, Amit Dadheech ${ }^{1}$, N. Kiran ${ }^{1}$, Prashant Bisen $^{1}$ and Santosh Kumar ${ }^{2}$ \\ ${ }^{1}$ Department of Plant Breeding and Genetics, Rajasthan College of Agriculture, Maharana Pratap \\ University of Agriculture and Technology, Udaipur- 313001, India \\ ${ }^{2}$ Department of Plant Breeding and Genetics, Dr. Rajendra Prasad Central Agricultural \\ University, Pusa, Samastipur, Bihar (848125), India \\ *Corresponding author
}

\begin{abstract}
Keywords
Maize, Diallel

analysis,

Combining ability

effects, GCA

variance, SCA

variance, Over

environments.

Article Info

Accepted:

04 September 2017

Available Online:

10 October 2017 harvest index exhibited high mean sum of squares due to GCA than SCA in $E_{1}$ environment while, in $\mathrm{E}_{2}$ environment they exhibited high mean sum of squares due to SCA than GCA. The estimates of SCA variance were of higher magnitude than GCA variance for all the traits in both the environments. In pooled analysis mean squares due to environment, GCA, SCA, GCA x E and SCA x E were significant for all the three characters except GCA $x$ E and SCA $x$ E for number of grain rows per ear. The estimates of GCA effects for grain yield per plant revealed that only three inbred lines viz., EIQ-106, EIQ-109 and EIQ-112 in $\mathrm{E}_{1}$ and only two inbreds; EIQ-112 and EIQ-109 in $\mathrm{E}_{2}$ showed positive significant GCA effects. The estimates of positive significant SCA effects for grain yield per plant were depicted by 20 and 17 hybrids in $E_{1}$ and $E_{2}$ environments respectively. The estimates of GCA effects revealed that good general combiner inbred lines for grain yield per plant were EIQ-112, EIQ-109 and EIQ-106 in $\mathrm{E}_{1}$ environment while, EIQ-109 and EIQ-112 in $\mathrm{E}_{2}$ environment. On the basis of mean and SCA effects out of total 45 hybrids, five hybrids which exhibited higher mean and significant positive SCA effects for grain yield per plant were EIQ-110 x EIQ-112, EIQ-107 x EIQ-109, EIQ-109 x EIQ-111, EIQ-105 x EIQ-112 and EIQ-105 x EIQ-114 in $E_{1}$ environment while hybrids EIQ-110 x EIQ-112, EIQ-105 x EIQ-112, EIQ-109 x EIQ-111, EIQ-107 $x$ EIQ-109, EIQ-105 x EIQ-113 in $\mathrm{E}_{2}$ environment. Among these the crosses EIQ-110 $\mathrm{x}$ EIQ-112, EIQ-107 x EIQ-109, EIQ-109 x EIQ-111, EIQ-105 x EIQ-112 were better performer in both the environment.
\end{abstract}

A B S T R A C T

Present study was conducted with 10 parents, their 45 hybrids along with 4 checks viz., Pratap QPM Hybrid-1, Vivek QPM-9, HQPM-1 and HQPM-5 to estimate GCA and SCA of parents and crosses respectively in diallel mating design (excluding reciprocal crosses) in two environments (Kharif 2014 and Rabi 2014-15) at the Instructional farm, Rajasthan College of Agriculture, MPUAT, Udaipur. Two characters viz., grain yield per plant and

\section{Introduction}

Maize is the third most widely distributed crop of the world after Rice and Wheat (Poehlman, 2006), being grown in diverse seasons and ecologies with highest production and productivity among food cereals. It is an important food, feed and industrial crop in India as well as other countries of the world which is believed to be originated in Southern 
Mexico or Northern Guatemala (Weatherwax, 1955). It has great yield potential and attained the leading position among the cereals based on production as well as productivity (Keskin et al., 2005). Every part of the plant has economic value; the grain, leaves, stalk, tassel are used to produce hundreds of food and non-food products (Haddadi et al., 2012). Maize ranks first in global cereal production of approximately 967 million tonnes in 2014 from 177 million hectare area with an average yield of 5.50 tonnes/hectare (Foreign Agriculture Services/USDA, Office of Global Analysis, 2014). In India, maize is cultivated on an area of 9.23 million ha with a production of 23.67 million tonnes and productivity of $2564 \mathrm{~kg} / \mathrm{ha}$ (Annual progress report, AICRP on Maize 2015: Indian Institute of Maize Research, New Delhi). To establish a sound basis for any breeding programme, breeders must have information on the nature of combining ability of parents, their behaviour and performance in hybrid combinations (Chawla and Gupta, 1984). Such knowledge of combining ability is essential for selection of suitable parents for hybridization and identification of promising hybrids for the development of improved varieties for a diverse agro-ecology (Alabi et al., 1987). One of the most informative methodology in this concern is diallel analysis system which is widely and extensively used for estimating the types of gene action. The two main genetic parameters of diallel analysis are GCA and SCA which are essential in developing breeding strategies. Combining ability is a powerful tool in identifying the best combiners for hybridization especially, when a large number of advance inbred lines are available and most promising ones are to be selected on the basis of their ability to give superior quality protein maize hybrids. General and specific combining ability are due to genes which are largely additive and dominance or epistatic effects respectively (Sprague and Tatum,
1942). Therefore, the present investigation was undertaken to study the general and specific combining ability of parents and crosses respectively over the environments for grain yield and yield contributing traits.

\section{Materials and Methods}

The materials of present investigation consisted of 10 inbred lines crossed in diallel design (Griffing, Model-I, Method-II) during Rabi 2013-14 to develop 45 half-diallel crosses (reciprocals excluded). These $45 \mathrm{~F}_{1}$ hybrids along with 10 parents (EIQ-105, EIQ-106, EIQ-107, EIQ-108, EIQ-109, EIQ-110, EIQ-111, EIQ-112, EIQ-113 and EIQ-114) and 4 checks viz., Pratap QPM Hybrid-1, Vivek QPM-9, HQPM-1 and HQPM-5 were evaluated for GCA and SCA of parents and crosses respectively in randomized block design (RBD) with three replications during Kharif 2014 and Rabi 2014-15. The materials were grown in a single row plot of four meter length, maintaining crop geometry of $60 \times 25 \mathrm{~cm}$. The observations were taken for yield and yield contributing traits viz., ear length $(\mathrm{cm})$, ear girth $(\mathrm{cm})$, no. of grain rows per ear, 100 grain weight $(\mathrm{g})$ harvest index $(\%)$ and grain yield per plant $(\mathrm{g})$. The combining ability analysis for individual environment as well as over environments was performed to determine the general and specific combining ability effects. The combining ability analysis of data for individual environment was carried out for both environments separately, using Griffing's method II, model I (1956). The combining ability analysis for over environments was carried out using the method suggested by Singh (1973 and 1979), which is an extension of Griffing's method II, model I (1956) to estimate the interactions of general and specific combining ability effects with environments, besides determining the significance of general and specific combining ability variance. 


\section{Results and Discussion}

Analysis of variance in individual environment revealed that mean sum of squares due to genotypes was significant for all the characters in both the environments (Table 2). Similar results were observed by Dar et al., (2015), Kumar et al., (2013) and Krupakar et al., (2013). By further partitioning of genotypic variance the mean square due to parents were significant for all the characters except for grain yield per plant which was non-significant in E2 environment. Mean squares due to crosses and mean sum of square due to parents $\mathrm{v} / \mathrm{s}$ crosses were significant for all the characters in both the environments. This result is in accordance with Lal and Kumar (2012). The Bartlett test revealed that error variance was homogenous for ear girth, number of grain rows per ear and harvest index. Therefore, pooled analysis was carried out for these characters only. The results of Bartlett test are comparable with the findings of Bacha et al., 2001. The pooled analysis revealed significant differences between the environments for the three characters. The mean sum of squares due to genotypes, parents, crosses, parents vs. crosses and parents $\mathrm{x}$ environments were also significant for all the three characters. Mean sum of squares due to genotypes $x$ environment interactions were significant for ear girth and harvest index, whereas for number of grain rows per ear it was nonsignificant, indicating influence of environments on the expression of this character. The mean sum of squares due to crosses $\mathrm{x}$ environments interaction were significant for ear girth and harvest index while non-significant for number of grain rows per ear (Table 3 ). The mean sum of squares for combining ability and estimated components of genetic variance for various characters are presented in (Table 4). The analysis of variance for combining ability revealed significant mean sum of squares due to GCA and SCA for all the traits, excepted mean sum of squares due to SCA for number of grain rows per year in E1 environment. These results suggested the existence of additive and non-additive gene actions for various traits in the material under study. The magnitude of mean sum of squares due to GCA was higher than SCA for ear length, ear girth and 100 grain weight in both the environments. In case of number of grain rows per ear the mean sum of squares due to SCA was higher than that of GCA in E1 environment while, mean sum of squares due to GCA was higher than SCA in E2 environment. Two characters viz., grain yield per plant and harvest index exhibited high mean sum of squares due to GCA than SCA in E1 environment (same result for grain yield per plant was observed by Moneam et al., 2009) while, in E2 environment they exhibited high mean sum of squares due to SCA than GCA. The estimates of SCA variance were of higher magnitude than GCA variance for all the traits in both the environments. In pooled analysis mean squares due to environment, GCA, SCA, GCA $x \mathrm{E}$ and SCA $\times \mathrm{E}$ were significant for all the three characters except GCA x E and SCA $\mathrm{x} E$ interactions for number of grain rows per ear (Table 5). Similar results for pooled mean squares due to GCA and SCA were observed by Nass et al., 2000; Aguiar et al., 2003; Qi et al., 2010; Alamerew and Warsi, 2015. The $\sigma 2$ SCA was higher than their corresponding $\sigma 2$ GCA for all the three characters. These results were also supported by the earlier findings of Vasal et al., (1992), Joshi et al., (1998) and Izhar and Chakraborty (2013). Similarly, $\sigma 2$ GCA x E were higher than the $\sigma 2$ SCA $x \mathrm{E}$ for all the three characters.

The combining ability analysis was performed to obtain information on selection of better parents and crosses for their further use in breeding programme. The estimates of gca effects of the parents and SCA effects of the 
hybrids for different traits are presented in (Tables 6 and 7). In case of ear length, out of ten inbred lines, three inbred lines in E1 viz., EIQ-109 (0.34) and EIQ-112 (0.75) and four inbred lines in E2 viz., EIQ-108 (0.35), EIQ109 (0.48), EIQ-112 (0.81) and EIQ-105 (0.88) exhibited positive significant GCA effects. Estimates of SCA effects revealed that out of forty- five hybrids, seventeen hybrids in E1 with the magnitude ranged from 0.85 (EIQ-109 x EIQ-111) to 3.51 (EIQ-106 $\mathrm{x}$ EIQ-114) and ten hybrids in E2 with the range from 1.14 (EIQ-106 $x$ EIQ-109 and EIQ -106 x EIQ-112) to 2.65 (EIQ-106 x EIQ-110) exhibited significant SCA effects in positive direction. For ear girth, two inbred lines viz., EIQ-109 (0.34) and EIQ-112 (0.75) in E1 and EIQ-105 (0.30) and EIQ109 (0.50) in E2 exhibited positive significant GCA effects. Estimates of SCA effects revealed that out of forty- five hybrids, eleven hybrids in E1 with the magnitude ranged from 0.96 (EIQ-108 x EIQ-113 and EIQ-113 x EIQ-114) to 2.25 (EIQ-105 x EIQ-112) and only four hybrids in E2 with the range from 0.71 (EIQ-111 x EIQ-114) to 0.99 (EIQ-106 $x$ EIQ-107) exhibited significant SCA effects in positive direction. Analysis of pool data for ear girth revealed that two inbreds viz., EIQ-
112 (0.24) and EIQ-109 (0.42) showed positive significant gca effects while, out of forty five, fifteen hybrids with the range 0.54 (EIQ-108 x EIQ-110 and EIQ-109 x EIQ113 ) to 1.26 (EIQ-110 x EIQ-112) expressed positive significant SCA effects. The estimates of positive significant GCA effects for number of grain rows per ear revealed that only one inbred line in both the environments with the magnitude 0.43 and 0.77 (EIQ-114) expressed positive significant GCA effects. The positive significant SCA effects for number of grain rows per ear was exhibited by six hybrids in E1 with the magnitude ranged from 1.13 (EIQ-105 x EIQ-110 and EIQ-106 x EIQ-114) to 1.52 (EIQ-108 x EIQ-109). In E2, only four hybrids viz., EIQ109 x EIQ-112 (1.16), EIQ-110 x EIQ-111 (1.32), EIQ-110 x EIQ-112 (1.49) and EIQ106 x EIQ-114 (2.21) exhibited positive significant SCA effects for number of grain rows per ear. Analysis of pooled data for number of grain rows per ear revealed that only one hybrid; EIQ-114 (0.60) depicted positive significant GCA effects. Among the hybrids, five hybrids with the magnitude ranged from 0.84 (EIQ-107 x EIQ-113) to 1.67 (EIQ-106 x EIQ-114) exhibited positive significant SCA effects.

Table.1 Estimates of GCA effects for yield and yield contributing traits in individual environment and over the environments

\begin{tabular}{|l|l|l|l|l|}
\hline \multirow{2}{*}{$\begin{array}{l}\text { S. } \\
\text { No. }\end{array}$} & \multicolumn{1}{|c|}{ Characters/Traits } & \multicolumn{3}{|c|}{ Good General Combiners } \\
\cline { 3 - 5 } & & \multicolumn{1}{|c|}{ E $_{\mathbf{1}}$} & \multicolumn{1}{c|}{ E $_{\mathbf{2}}$} & \multicolumn{1}{c|}{ Pool } \\
\hline $\mathbf{1}$ & Ear Length & $\begin{array}{l}\text { EIQ-112, EIQ- } \\
110 \text { and EIQ-107 }\end{array}$ & $\begin{array}{l}\text { EIQ-105, EIQ-112, } \\
\text { EIQ-109 and EIQ-108 }\end{array}$ & - \\
\hline $\mathbf{2}$ & Ear Girth & $\begin{array}{l}\text { EIQ-112 and } \\
\text { EIQ-109 }\end{array}$ & EIQ-105 and EIQ-109 & $\begin{array}{l}\text { EIQ-112 and } \\
\text { EIQ-109 }\end{array}$ \\
\hline $\mathbf{3}$ & No. of Grain Rows per Ear & EIQ-114 & EIQ-114 & EIQ-114 \\
\hline $\mathbf{4}$ & $\mathbf{1 0 0}$ Grain Weight & $\begin{array}{l}\text { EIQ-112, EIQ- } \\
111, \text { and EIQ-106 }\end{array}$ & $\begin{array}{l}\text { EIQ-106, EIQ-113, } \\
\text { EIQ-105 and EIQ-110 }\end{array}$ & - \\
\hline $\mathbf{5}$ & Harvest Index & $\begin{array}{l}\text { EIQ-106, EIQ- } \\
109 \text { and EIQ-112 }\end{array}$ & $\begin{array}{l}\text { EIQ-106, EIQ-113, } \\
\text { EIQ-110 and EIQ-109 }\end{array}$ & $\begin{array}{l}\text { EIQ-106, EIQ- } \\
109 \text { and EIQ-113 }\end{array}$ \\
\hline $\mathbf{6}$ & Grain Yield per Plant & $\begin{array}{l}\text { EIQ-112, EIQ- } \\
109 \text { and EIQ-106 }\end{array}$ & EIQ-109 and EIQ-112 & - \\
\hline
\end{tabular}


Table.2 Mean squares for different characters in Maize (Zea mays L.) environment wise

\begin{tabular}{|c|c|c|c|c|c|c|c|c|c|}
\hline \multirow[t]{3}{*}{$\overline{\mathbf{S N}}$} & \multirow[t]{3}{*}{ Characters } & \multirow[t]{3}{*}{ Env } & \multicolumn{6}{|c|}{ Source } & \multirow[t]{2}{*}{ Bartlett } \\
\hline & & & Rep & Genotype & Parent & Crosses & $\begin{array}{l}\text { Parents vs } \\
\text { crosses }\end{array}$ & Error & \\
\hline & & & [2] & {$[54]$} & {$[9]$} & {$[44]$} & [1] & {$[108]$} & [1] \\
\hline \multirow[t]{2}{*}{1} & \multirow[t]{2}{*}{ Ear length $(\mathrm{cm})$} & 1 & 1.38788 & $11.9556 * *$ & $11.7185 * *$ & $10.0526 * *$ & $97.8185 * *$ & 0.536027 & \multirow[b]{2}{*}{$14.87 * *$} \\
\hline & & 2 & $4.22769 *$ & $8.46996^{* *}$ & $7.18967 * *$ & $5.75756 * *$ & $139.338 * *$ & 1.13552 & \\
\hline \multirow[t]{2}{*}{2} & \multirow[t]{2}{*}{ Ear girth $(\mathrm{cm})$} & 1 & 1.16515 & $4.16818 * *$ & $3.7787 * *$ & $2.9766^{* *}$ & $60.1031 * *$ & 0.55867 & \multirow[b]{2}{*}{2.37689} \\
\hline & & 2 & 0.878571 & $1.68896 * *$ & $2.97052 * *$ & $0.675731^{*}$ & $34.7372 * *$ & 0.415013 & \\
\hline \multirow[t]{2}{*}{3} & \multirow{2}{*}{$\begin{array}{l}\text { Number of grain rows per } \\
\text { ear }\end{array}$} & 1 & 0.169697 & $2.70438 * *$ & $3.68889 * *$ & $2.23973 * *$ & $14.2882 * *$ & 1.13266 & \multirow[b]{2}{*}{0.0252574} \\
\hline & & 2 & 0.678788 & $3.03659 * *$ & $4.74074 * *$ & $2.04983 * *$ & $31.1165 * *$ & 1.09854 & \\
\hline \multirow[t]{2}{*}{4} & \multirow[t]{2}{*}{ 100-Grain weight $(\mathrm{g})$} & 1 & 1.57276 & $27.5754 * *$ & $8.11111^{* *}$ & $28.0809 * *$ & $180.511 * *$ & 0.82675 & \multirow[b]{2}{*}{$16.8673 * *$} \\
\hline & & 2 & $6.65486^{*}$ & $17.2658 * *$ & $11.2576^{* *}$ & $17.2588 * *$ & $71.6491 * *$ & 1.84128 & \\
\hline \multirow[t]{2}{*}{5} & \multirow[t]{2}{*}{ Grain yield per plant (g) } & 1 & 15.8061 & $982.046 * *$ & $127.482 * *$ & $853.212 * *$ & $14341.8^{* *}$ & 10.411 & \multirow[b]{2}{*}{$65.9651 * *$} \\
\hline & & 2 & 69.1869 & $665.872 * *$ & 56.3824 & $447.078 * *$ & $15778.2 * *$ & 53.8692 & \\
\hline \multirow[t]{2}{*}{6} & \multirow[t]{2}{*}{ Harvest index (\%) } & 1 & 1.3373 & $30.8761 * *$ & $18.3143 * *$ & $27.902 * *$ & $274.792 * *$ & 2.55375 & \multirow[b]{2}{*}{0.484708} \\
\hline & & 2 & $7.05377^{*}$ & $31.5175^{* *}$ & $19.7769 * *$ & $23.4901 * *$ & $490.392 * *$ & 2.23341 & \\
\hline
\end{tabular}

*, ** Significant at 5 and 1 per cent respectively (Model I)

Table.3 Pooled mean squares for Ear girth, Number of grain rows per ear and Harvest index in Maize (Zea mays L.)

\begin{tabular}{|c|c|c|c|c|c|c|c|c|c|c|c|c|c|}
\hline \multirow[t]{3}{*}{ SN } & \multirow[t]{3}{*}{ Characters } & \multicolumn{11}{|c|}{ Source } & \multirow[t]{2}{*}{ Bartlett } \\
\hline & & Env & Rep/Env & Genotype & Parents & Crosses & $\begin{array}{c}\text { Parents } \\
\text { vs crosses }\end{array}$ & GxE & $\begin{array}{c}\text { Parent } x \\
\text { Env }\end{array}$ & $\begin{array}{c}\text { Crosses } x \\
E\end{array}$ & \begin{tabular}{|c|} 
Parents vs \\
Crosses x E
\end{tabular} & $\begin{array}{c}\text { Pool } \\
\text { Error }\end{array}$ & \\
\hline & & {$[1]$} & [4] & {$[54]$} & [9] & {$[44]$} & [1] & [54] & [9] & [44] & {$[1]$} & [216] & [1] \\
\hline 1 & $\begin{array}{l}\text { Ear girth } \\
(\mathrm{cm})\end{array}$ & $22.5688 * *$ & 1.02186 & $4.17592 * *$ & $4.67498 * *$ & $2.05255^{* *}$ & $93.1128 * *$ & $1.68123 * *$ & $2.07424 * *$ & $1.59979 * *$ & 1.72755 & 0.486842 & 2.37689 \\
\hline 2 & $\begin{array}{l}\text { Number of } \\
\text { grain rows } \\
\text { per ear }\end{array}$ & $87.5758 * *$ & 0.424242 & $4.23928 * *$ & $5.25185 * *$ & $3.13333 * *$ & $43.7879 * *$ & 1.50168 & $3.17778 * *$ & 1.15623 & 1.61684 & 1.1156 & 0.0252574 \\
\hline 3 & $\begin{array}{l}\text { Harvest } \\
\text { index }(\%)\end{array}$ & $178.118 * *$ & 4.19554 & $52.7503 * *$ & $28.8107 * *$ & $41.8076^{* * *}$ & $749.683 * *$ & $9.64332 * *$ & $9.28043 * *$ & $9.58442 * *$ & $15.501^{*}$ & 2.39358 & 0.484708 \\
\hline
\end{tabular}

*,** Significant at 5 and 1 per cent respectively (Model I) 
Table.4 Combining ability mean squares and EMS for different characters in maize (Zea mays L.) environment wise

\begin{tabular}{|c|c|c|c|c|c|c|c|}
\hline \multirow[t]{3}{*}{$\mathbf{S N}$} & \multirow[t]{3}{*}{ Characters } & \multirow[t]{3}{*}{ Env } & \multicolumn{3}{|c|}{ Source } & \multicolumn{2}{|c|}{ Var Model I } \\
\hline & & & GCA & SCA & Error & GCA & SCA \\
\hline & & & [9] & {$[45]$} & {$[108]$} & & \\
\hline \multirow[t]{2}{*}{6} & \multirow[t]{2}{*}{ Ear length $(\mathrm{cm})$} & 1 & $4.70535 * *$ & $3.84115 * *$ & 0.178676 & 1.6975 & 82.4057 \\
\hline & & 2 & $5.46514 * *$ & $2.29494 * *$ & 0.378507 & 1.90749 & 43.1198 \\
\hline \multirow[t]{2}{*}{7} & \multirow[t]{2}{*}{ Ear girth $(\mathrm{cm})$} & 1 & $1.58426^{* *}$ & $1.35042 * *$ & 0.186223 & 0.524263 & 26.1944 \\
\hline & & 2 & $0.760446^{* *}$ & $0.523487 * *$ & 0.138338 & 0.233291 & 8.66586 \\
\hline \multirow[t]{2}{*}{8} & \multirow[t]{2}{*}{ Number of grain rows per ear } & 1 & 0.46749 & $0.988253 * *$ & 0.377553 & 0.0337262 & 13.7407 \\
\hline & & 2 & $1.65267 * *$ & $0.8841^{* *}$ & 0.36618 & 0.482435 & 11.6532 \\
\hline \multirow[t]{2}{*}{9} & \multirow[t]{2}{*}{ 100-grain weight (g) } & 1 & $10.7448 * *$ & $8.88116^{* *}$ & 0.275583 & 3.92597 & 193.626 \\
\hline & & 2 & 6.03394** & $5.69953 * *$ & 0.613761 & 2.03257 & 114.43 \\
\hline \multirow[t]{2}{*}{$\mathbf{1 0}$} & \multirow[t]{2}{*}{ Grain yield per plant (g) } & 1 & $441.615 * *$ & $304.495 * *$ & 3.47033 & 164.304 & 6773.06 \\
\hline & & 2 & $204.363 * *$ & $225.476^{* *}$ & 17.9564 & 69.9024 & 4669.19 \\
\hline \multirow[t]{2}{*}{11} & \multirow[t]{2}{*}{ Harvest index (\%) } & 1 & $10.7325^{* *}$ & $10.204 * *$ & 0.851251 & 3.70548 & 210.437 \\
\hline & & 2 & $4.70446 * *$ & $11.666^{* *}$ & 0.744469 & 1.485 & 245.735 \\
\hline
\end{tabular}

*, ** Significant at 5 and 1 per cent respectively (Model I)

Table.5 Combining ability mean square and EMS over the environments for Ear girth, Number of grain rows per ear and Harvest index

\begin{tabular}{|c|c|c|c|c|c|c|c|c|c|c|c|c|}
\hline \multirow{3}{*}{$\begin{array}{l}\mathbf{S} \\
\mathbf{N}\end{array}$} & \multirow{3}{*}{$\begin{array}{c}\text { Characte } \\
\text { rs }\end{array}$} & \multicolumn{6}{|c|}{ Source } & Bartlett & \multicolumn{4}{|c|}{ Variance Model I } \\
\hline & & Env & GCA & SCA & GCA $\times$ E & SCA $\times \mathbf{E}$ & Pool Error & & GCA & SCA & $\begin{array}{c}\text { GCA } x \\
\text { E }\end{array}$ & SCA $\times$ E \\
\hline & & [1] & [9] & [45] & [9] & [45] & [216] & [1] & & & & \\
\hline 1 & $\begin{array}{c}\text { Ear girth } \\
(\mathrm{cm})\end{array}$ & $2.50764 * *$ & $\begin{array}{c}1.11008 * \\
*\end{array}$ & $\begin{array}{c}1.44834 * \\
*\end{array}$ & $1.23463 * *$ & $0.425565 * *$ & 0.162281 & 2.37689 & 0.0426429 & $\begin{array}{c}0.35542 \\
4 \\
\end{array}$ & $\begin{array}{c}28.936 \\
4\end{array}$ & 0.80426 \\
\hline 2 & $\begin{array}{c}\text { Number } \\
\text { of grain } \\
\text { rows per } \\
\text { ear }\end{array}$ & $9.73064 * *$ & $\begin{array}{c}1.53992 * \\
*\end{array}$ & $\begin{array}{c}1.38773 * \\
*\end{array}$ & 0.580247 & 0.484624 & 0.371867 & $\begin{array}{c}0.025257 \\
4\end{array}$ & 0.17016 & $\begin{array}{c}0.43801 \\
9\end{array}$ & $\begin{array}{c}22.856 \\
9\end{array}$ & 0.156285 \\
\hline 3 & $\begin{array}{c}\text { Harvest } \\
\text { Index } \\
(\%)\end{array}$ & $19.7909 * *$ & $\begin{array}{c}11.7619 * \\
*\end{array}$ & $\begin{array}{c}18.7474 * \\
*\end{array}$ & $3.67505 * *$ & $3.12262 * *$ & 0.79786 & 0.484708 & 0.345329 & 4.11153 & $\begin{array}{c}403.86 \\
5\end{array}$ & 2.15789 \\
\hline
\end{tabular}

*, ** Significant at 5 and 1 per cent respectively (Model I) 
Table.6 GCA and SCA effects for Ear length (cm), 100-grain weight $(\mathrm{g})$ and Grain yield per plant $(\mathrm{g})$

\begin{tabular}{|c|c|c|c|c|c|c|c|}
\hline \multirow[t]{2}{*}{ SN } & \multirow[t]{2}{*}{ Genotype } & \multicolumn{2}{|c|}{ Ear length $(\mathrm{cm})$} & \multicolumn{2}{|c|}{$\begin{array}{l}\text { 100-grain weight } \\
\text { (g) }\end{array}$} & \multicolumn{2}{|c|}{$\begin{array}{c}\text { Grain yield per plant } \\
\text { (g) }\end{array}$} \\
\hline & & E1 & E2 & E1 & E2 & E1 & E2 \\
\hline 1 & EIQ-105 & -0.04 & $0.88 * *$ & $-0.98 * *$ & $0.61 * *$ & $-2.41 * *$ & -1.40 \\
\hline 2 & EIQ-106 & -0.09 & $-0.35^{*}$ & $0.45^{* *}$ & $0.83 * *$ & $3.05 * *$ & 2.23 \\
\hline 3 & EIQ-107 & $0.38 * *$ & -0.09 & 0.05 & $-0.95 * *$ & $-4.11 * *$ & $-2.54 *$ \\
\hline 4 & EIQ-108 & $-0.24 *$ & $0.35 *$ & $-1.80 * *$ & $-0.89 * *$ & $-9.97 * *$ & $-6.32 * *$ \\
\hline 5 & EIQ-109 & 0.19 & $0.48 * *$ & 0.16 & 0.12 & $8.89 * *$ & $6.79 * *$ \\
\hline 6 & EIQ-110 & $0.51 * *$ & 0.01 & -0.09 & $0.50 *$ & $-1.38 * *$ & 1.23 \\
\hline 7 & EIQ-111 & $-0.30 *$ & $-0.47 * *$ & $1.47 * *$ & 0.21 & $-2.61 * *$ & $-3.65 * *$ \\
\hline 8 & EIQ-112 & $1.17 * *$ & $0.81 * *$ & $1.17 * *$ & -0.04 & $10.46^{* *}$ & $6.01 * *$ \\
\hline 9 & EIQ-113 & $-0.38 * *$ & $-1.37 * *$ & -0.26 & $0.64 * *$ & -0.36 & -1.15 \\
\hline 10 & EIQ-114 & $-1.19 * *$ & -0.25 & -0.19 & $-1.03^{* *}$ & $-1.58 * *$ & -1.19 \\
\hline 11 & EIQ-106 x EIQ-105 & 0.69 & -0.46 & $-1.36 * *$ & -0.10 & -2.63 & 0.37 \\
\hline 12 & EIQ-107 x EIQ-105 & 0.39 & 0.21 & $2.37 * *$ & -0.65 & $-7.48 * *$ & -4.19 \\
\hline 13 & EIQ-108 x EIQ-105 & $1.01 *$ & 0.74 & -0.94 & -1.13 & -0.28 & -3.08 \\
\hline 14 & EIQ-109 x EIQ-105 & $-2.58 * *$ & $1.81 * *$ & $-1.40 * *$ & -1.39 & $-12.81 * *$ & $-10.52 * *$ \\
\hline 15 & EIQ-110 x EIQ-105 & $-1.07 * *$ & 0.31 & $-5.99 * *$ & 0.73 & $4.80 * *$ & 6.37 \\
\hline 16 & EIQ-111 x EIQ-105 & $0.90 *$ & -0.07 & -0.71 & 0.52 & $-7.31 * *$ & -2.41 \\
\hline 17 & EIQ-112 x EIQ-105 & $1.76^{* *}$ & -0.59 & $2.59 * *$ & 1.11 & $25.95 * *$ & $26.59 * *$ \\
\hline 18 & EIQ-113 x EIQ-105 & $1.15^{* *}$ & $1.60 * *$ & $4.18 * *$ & 0.59 & $21.77 * *$ & $21.75 * *$ \\
\hline 19 & EIQ-114 x EIQ-105 & 0.12 & -0.43 & $3.11 * *$ & $2.09 * *$ & $25.66 * *$ & $17.12 * *$ \\
\hline 20 & EIQ-107 x EIQ-106 & 0.61 & $1.51 * *$ & $2.78 * *$ & $2.80 * *$ & $6.40 * *$ & $8.17 *$ \\
\hline 21 & EIQ-108 x EIQ-106 & -0.26 & 0.68 & -0.54 & $2.74 * *$ & 0.59 & $10.28 * *$ \\
\hline 22 & EIQ-109 x EIQ-106 & 0.47 & $1.14 *$ & $3.17 * *$ & $2.40 * *$ & $15.06 * *$ & 7.17 \\
\hline 23 & EIQ-110 x EIQ-106 & $1.99 * *$ & $2.65 * *$ & $2.42 * *$ & $3.60 * *$ & $11.01 * *$ & $14.06 * *$ \\
\hline 24 & EIQ-111 x EIQ-106 & 0.46 & 0.19 & 0.70 & -0.28 & $-8.44 * *$ & -0.72 \\
\hline 25 & EIQ - $112 \times$ EIQ - 106 & $2.65^{* *}$ & $1.14 *$ & $1.15^{*}$ & $2.89 * *$ & $18.99 * *$ & $7.95 *$ \\
\hline 26 & EIQ-113 x EIQ-106 & -0.29 & 0.80 & -0.25 & $-1.96 * *$ & $8.31^{* *}$ & 5.78 \\
\hline 27 & EIQ-114 x EIQ-106 & $3.51 * *$ & 1.07 & $-1.82 * *$ & -0.13 & $-6.46 * *$ & -2.51 \\
\hline 28 & EIQ - 108 x EIQ - 107 & 0.60 & -0.18 & 0.19 & -1.32 & 2.74 & -4.61 \\
\hline 29 & EIQ-109 x EIQ-107 & $3.50 * *$ & 0.48 & $4.07 * *$ & $-1.99 * *$ & $31.22 * *$ & $21.95 * *$ \\
\hline 30 & EIQ-110 x EIQ-107 & $-1.49 * *$ & $-1.24 *$ & $1.35 * *$ & 0.29 & $-6.51 * *$ & -0.83 \\
\hline
\end{tabular}




\begin{tabular}{|c|c|c|c|c|c|c|c|c|}
\hline \multirow[t]{2}{*}{ SN } & \multirow{2}{*}{\multicolumn{2}{|c|}{ Genotype }} & \multicolumn{2}{|c|}{ Ear length (cm) } & \multicolumn{2}{|c|}{$\begin{array}{c}\text { 100-grain weight } \\
\text { (g) }\end{array}$} & \multicolumn{2}{|c|}{$\begin{array}{c}\text { Grain yield per plant } \\
\text { (g) }\end{array}$} \\
\hline & & & E1 & $\mathbf{E 2}$ & E1 & E2 & E1 & $\mathbf{E 2}$ \\
\hline 31 & \multicolumn{2}{|c|}{ EIQ-111 x EIQ-107 } & $-0.85^{*}$ & 0.87 & 0.60 & $-1.92 * *$ & -1.95 & -0.61 \\
\hline 32 & \multicolumn{2}{|c|}{ EIQ-112 x EIQ-107 } & -0.49 & 0.65 & $1.89 * *$ & $-2.00 * *$ & $6.31 * *$ & 1.73 \\
\hline 33 & \multicolumn{2}{|c|}{ EIQ-113 x EIQ-107 } & $-1.10 * *$ & 0.61 & $-2.35 * *$ & $2.82 * *$ & $9.13 * *$ & $9.23 *$ \\
\hline 34 & \multicolumn{2}{|c|}{ EIQ-114 x EIQ-107 } & $1.21 * *$ & 0.92 & $-2.25^{* *}$ & -0.85 & 2.36 & $9.93 *$ \\
\hline 35 & \multicolumn{2}{|c|}{ EIQ-109 x EIQ-108 } & $1.46^{* *}$ & 0.11 & $2.92 * *$ & $-2.06 * *$ & -0.59 & $10.73 * *$ \\
\hline 36 & \multicolumn{2}{|c|}{ EIQ-110 x EIQ-108 } & $1.14 * *$ & $2.02 * *$ & 0.67 & $-2.77 * *$ & $7.02 * *$ & 7.28 \\
\hline 37 & \multicolumn{2}{|c|}{ EIQ-111 x EIQ - 108} & $-3.39 * *$ & -1.10 & $-1.55 * *$ & -0.31 & 0.58 & 3.84 \\
\hline 38 & \multicolumn{2}{|c|}{ EIQ-112 x EIQ-108 } & -0.53 & -0.42 & -0.10 & $2.61 * *$ & $-8.16^{* *}$ & -0.83 \\
\hline 39 & \multicolumn{2}{|c|}{ EIQ-113 x EIQ-108 } & $2.69 * *$ & $1.37 *$ & $1.84 * *$ & $3.17 * *$ & -1.01 & -4.00 \\
\hline 40 & \multicolumn{2}{|c|}{ EIQ-114 x EIQ-108 } & $-2.50 * *$ & $1.35^{*}$ & $-1.23 *$ & -1.16 & $-4.12 *$ & 2.04 \\
\hline 41 & \multicolumn{2}{|c|}{ EIQ-110 x EIQ-109 } & -0.46 & 0.51 & $-2.96 * *$ & $6.06 * *$ & $-23.51 * *$ & $-17.16^{* *}$ \\
\hline 42 & \multicolumn{2}{|c|}{ EIQ-111 x EIQ-109 } & $0.85^{*}$ & -0.41 & $-1.01 *$ & -0.99 & $28.72 * *$ & $23.73 * *$ \\
\hline 43 & \multicolumn{2}{|c|}{ EIQ-112 x EIQ-109 } & $2.54 * *$ & 1.01 & $-1.22 *$ & 0.60 & $20.65 * *$ & $9.39 *$ \\
\hline 44 & \multicolumn{2}{|c|}{ EIQ-113 x EIQ-109 } & $1.43 * *$ & 0.33 & 0.38 & 1.08 & $15.47 * *$ & $14.56 * *$ \\
\hline 45 & \multicolumn{2}{|c|}{ EIQ-114 x EIQ-109 } & 0.74 & 0.21 & $1.81 * *$ & 0.08 & 0.02 & -4.40 \\
\hline 46 & \multicolumn{2}{|c|}{ EIQ-111 x EIQ-110 } & $-0.81 *$ & $1.50 * *$ & $-1.10^{*}$ & $-1.87 *$ & -2.01 & -2.38 \\
\hline 47 & \multicolumn{2}{|c|}{ EIQ-112 x EIQ-110 } & $2.56^{* *}$ & 0.45 & $7.86 * *$ & -0.28 & $34.59 * *$ & $27.95 * *$ \\
\hline 48 & \multicolumn{2}{|c|}{ EIQ-113 x EIQ-110 } & -0.22 & -0.29 & -0.38 & $-2.80 * *$ & $4.41 *$ & $7.78 *$ \\
\hline 49 & \multicolumn{2}{|c|}{ EIQ-114 x EIQ-110 } & $0.92 *$ & 0.85 & $-3.45 * *$ & -0.97 & $-7.70 * *$ & -1.18 \\
\hline 50 & \multicolumn{2}{|c|}{ EIQ-112 x EIQ-111 } & $-0.97 *$ & 1.07 & 0.47 & 1.34 & $-15.85^{* *}$ & $-8.16^{*}$ \\
\hline 51 & \multicolumn{2}{|c|}{ EIQ-113 x EIQ-111 } & -0.25 & $-1.98 * *$ & -0.10 & $2.49 * *$ & 3.30 & -6.33 \\
\hline 52 & \multicolumn{2}{|c|}{ EIQ-114 x EIQ-111 } & 0.06 & -0.44 & $8.00 * *$ & 0.16 & $21.86 * *$ & $12.71 * *$ \\
\hline 53 & \multicolumn{2}{|c|}{ EIQ-113 x EIQ-112 } & $-0.89^{*}$ & -0.43 & $-2.80 * *$ & 1.08 & $-12.44 * *$ & -6.66 \\
\hline 54 & EIQ-114 & EIQ-112 & 0.58 & 1.01 & -0.04 & $2.58 * *$ & $-4.88 * *$ & 2.04 \\
\hline 55 & EIQ-114 & EIQ-113 & $-1.53 * *$ & $-1.63 * *$ & $1.22 *$ & $-2.94 * *$ & $4.94^{* *}$ & -2.46 \\
\hline Stan & lard error & & & & & & & \\
\hline & $\mathrm{Gi}$ & 0.12 & 0.17 & 0.14 & & & 0.51 & 1.16 \\
\hline & $\mathrm{Gi}-\mathrm{Gj}$ & 0.17 & 0.25 & 0.21 & & & 0.76 & 1.73 \\
\hline & Sii & 0.35 & 0.51 & 0.43 & & & 1.54 & 3.50 \\
\hline & $\mathrm{Sij}$ & 0.39 & 0.57 & 0.48 & & & 1.72 & 3.90 \\
\hline & Sij-ik & 0.57 & 0.83 & 0.71 & & & 2.52 & 5.74 \\
\hline & Sij-Skl & 0.55 & 0.79 & 0.68 & & & 2.40 & 5.47 \\
\hline
\end{tabular}


Table.7 GCA and SCA effects for Ear girth (cm), No. of grain rows per ear and Harvest Index

\begin{tabular}{|c|c|c|c|c|c|c|c|c|c|c|}
\hline \multirow[t]{2}{*}{$\mathbf{S N}$} & \multirow[t]{2}{*}{ Genotype } & \multicolumn{3}{|c|}{ Ear girth (cm) } & \multicolumn{3}{|c|}{ Number of grain rows per ear } & \multicolumn{3}{|c|}{ Harvest index (\%) } \\
\hline & & E1 & E2 & Pool & E1 & E2 & Pool & E1 & E2 & Pool \\
\hline 1 & EIQ-105 & $-0.56 * *$ & $0.30 * *$ & -0.13 & -0.18 & 0.32 & 0.07 & -0.45 & 0.06 & -0.20 \\
\hline 2 & EIQ-106 & -0.04 & -0.16 & -0.10 & -0.12 & $-0.57 * *$ & $-0.34 *$ & $1.13 * *$ & $0.72 * *$ & $0.93 * *$ \\
\hline 3 & EIQ-107 & -0.03 & -0.05 & -0.04 & 0.04 & 0.16 & 0.10 & $-1.42 * *$ & $-0.93 * *$ & $-1.18 *$ \\
\hline 4 & EIQ-108 & $-0.24 *$ & -0.10 & $-0.17 *$ & -0.07 & -0.29 & -0.18 & $-1.62 * *$ & $-0.56^{*}$ & $-1.09 *$ \\
\hline 5 & EIQ-109 & $0.34 * *$ & $0.50 * *$ & $0.42 * *$ & -0.01 & 0.16 & 0.07 & $0.93 * *$ & $0.52 *$ & $0.72 * *$ \\
\hline 6 & EIQ-110 & $-0.34 * *$ & $-0.33 * *$ & $-0.33^{*}$ & -0.18 & -0.18 & -0.18 & -0.20 & $0.65 * *$ & 0.22 \\
\hline 7 & EIQ-111 & 0.14 & -0.05 & 0.04 & -0.18 & -0.07 & -0.12 & 0.19 & $-0.55^{*}$ & -0.18 \\
\hline 8 & EIQ-112 & $0.75 * *$ & $-0.26 *$ & $0.24 * *$ & 0.21 & -0.23 & -0.01 & $0.87 * *$ & $-0.61 *$ & 0.13 \\
\hline 9 & EIQ-113 & 0.05 & 0.09 & 0.07 & 0.04 & -0.07 & -0.01 & 0.46 & $0.67 * *$ & $0.57 * *$ \\
\hline 10 & EIQ-114 & -0.07 & 0.06 & -0.01 & $0.43 *$ & $0.77 * *$ & $0.60 * *$ & 0.11 & 0.02 & 0.07 \\
\hline 11 & EIQ-106 x EIQ-105 & 0.71 & 0.28 & 0.49 & 1.08 & 0.66 & $0.87 *$ & 0.42 & -0.33 & 0.04 \\
\hline 12 & EIQ-107 x EIQ-105 & 0.53 & $0.87 *$ & $0.70 * *$ & -0.42 & 0.60 & 0.09 & -0.01 & $2.61 * *$ & $1.30 *$ \\
\hline 13 & $\begin{array}{l}\text { EIQ - } 108 \times \text { EIQ - } \\
105\end{array}$ & 0.57 & -0.29 & 0.14 & 0.35 & 0.38 & 0.37 & $-2.69 * *$ & $-3.49 * *$ & $-3.09 * *$ \\
\hline 14 & EIQ-109 x EIQ-105 & $-0.85^{*}$ & -0.25 & $-0.55 *$ & 0.30 & -0.07 & 0.12 & $-3.90 * *$ & $-2.07 *$ & $-2.99 * *$ \\
\hline 15 & EIQ-110 x EIQ-105 & $-0.84 *$ & 0.15 & -0.34 & $1.13 *$ & 0.27 & 0.70 & 0.90 & 1.08 & 0.99 \\
\hline 16 & EIQ-111 x EIQ-105 & $-0.97 *$ & -0.36 & $-0.67 *$ & -0.20 & -0.51 & -0.36 & -1.04 & 0.80 & -0.12 \\
\hline 17 & EIQ-112 x EIQ-105 & $2.25 * *$ & -0.43 & $0.91 * *$ & 0.74 & -0.34 & 0.20 & $3.39 * *$ & 1.14 & $2.26 * *$ \\
\hline 18 & EIQ-113 x EIQ-105 & 0.44 & 0.03 & 0.23 & -0.42 & 0.16 & -0.13 & $9.11 * *$ & $9.81 * *$ & $9.46 * *$ \\
\hline 19 & EIQ-114 x EIQ-105 & 0.73 & 0.36 & $0.55^{*}$ & 0.52 & -0.68 & -0.08 & $3.24 * *$ & $1.86^{*}$ & $2.55 * *$ \\
\hline 20 & EIQ-107 x EIQ-106 & -0.16 & $0.99 * *$ & 0.42 & -0.48 & 0.82 & 0.17 & $4.68 * *$ & $6.26^{* *}$ & $5.47 * *$ \\
\hline 21 & EIQ-108 x EIQ-106 & -0.78 & 0.34 & -0.22 & 0.30 & -0.07 & 0.12 & -1.12 & $2.01 *$ & 0.45 \\
\hline 22 & EIQ-109 x EIQ-106 & $1.47 * *$ & -0.06 & $0.70 * *$ & 0.24 & 0.16 & 0.20 & 1.16 & -1.44 & -0.14 \\
\hline 23 & EIQ-110 x EIQ-106 & 0.65 & $0.97 * *$ & $0.81 * *$ & -0.26 & 0.49 & 0.12 & $1.78 *$ & $4.18 * *$ & $2.98 * *$ \\
\hline 24 & EIQ-111 x EIQ-106 & -0.16 & 0.23 & 0.03 & 1.08 & 0.38 & 0.73 & $-3.56 * *$ & $-2.54 * *$ & $-3.05 * *$ \\
\hline 25 & EIQ-112 x EIQ-106 & $1.07 * *$ & 0.43 & $0.75 * *$ & -0.65 & -0.12 & -0.38 & $2.20 *$ & 1.03 & $1.61 * *$ \\
\hline 26 & EIQ-113 x EIQ-106 & 0.09 & 0.18 & 0.14 & 0.85 & 0.38 & 0.62 & $2.29 * *$ & $2.02 *$ & $2.15 * *$ \\
\hline 27 & EIQ-114 x EIQ-106 & $1.39 * *$ & 0.65 & $1.02 * *$ & $1.13 *$ & $2.21 * *$ & $1.67 * *$ & -1.48 & -0.12 & -0.80 \\
\hline 28 & EIQ-108 x EIQ-107 & 0.21 & 0.26 & 0.23 & $-1.20 *$ & 0.55 & -0.33 & -1.11 & $-2.50 * *$ & $-1.80 * *$ \\
\hline 29 & EIQ-109 x EIQ-107 & $2.12 * *$ & 0.26 & $1.19 * *$ & $1.41 *$ & -0.57 & 0.42 & $4.14 * *$ & -0.69 & $1.72 * *$ \\
\hline 30 & EIQ-110 x EIQ-107 & 0.47 & 0.40 & 0.43 & 0.24 & -0.23 & 0.01 & $-2.55^{* *} *$ & -1.47 & $-2.01 * *$ \\
\hline
\end{tabular}




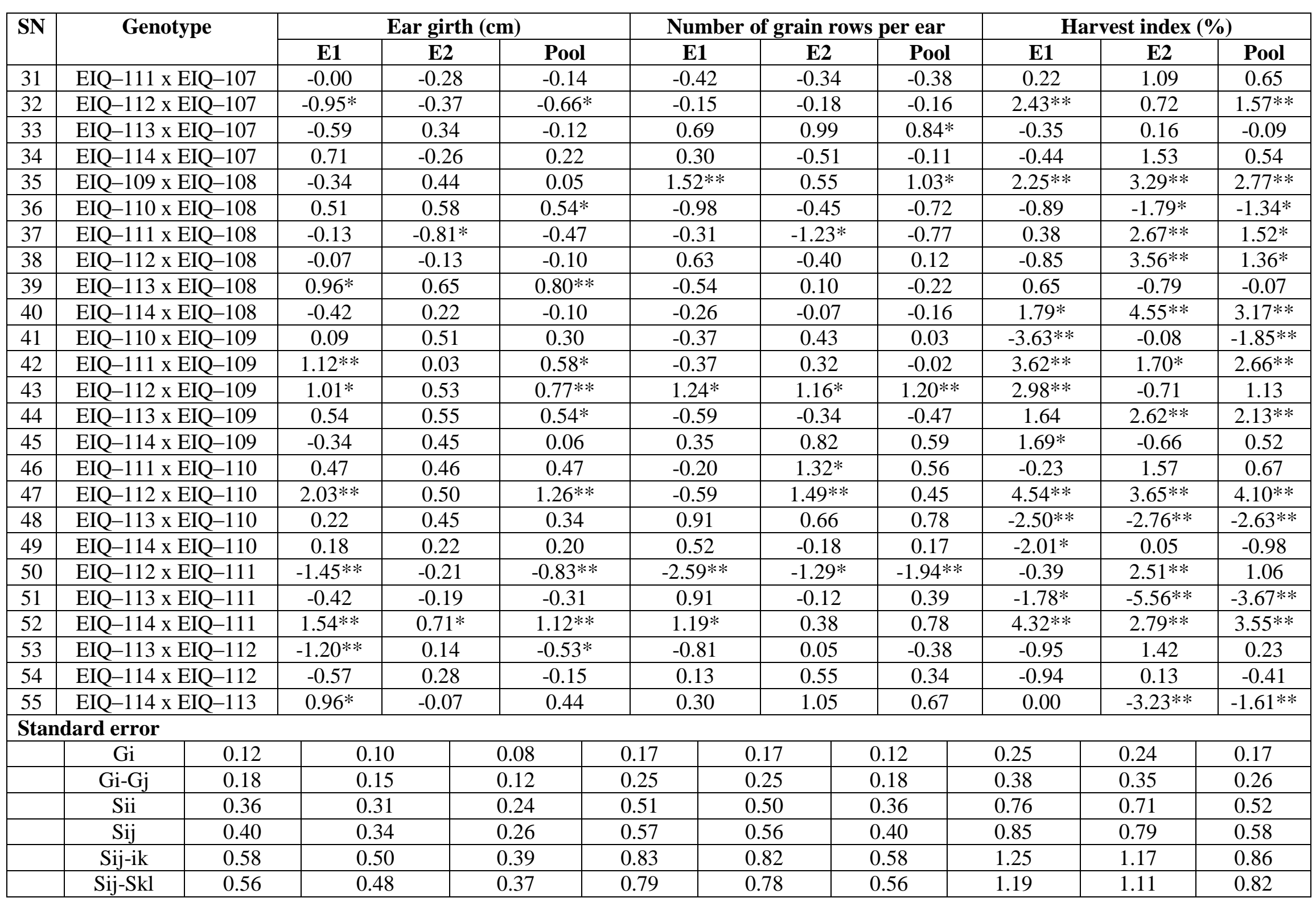


The estimates of positive significant GCA effects for 100-grain weight revealed that among the ten inbred lines, only three inbred lines viz., EIQ-106 (0.45), EIQ-112 (1.17) and EIQ-111 (1.47) in E1 and only four inbred lines viz., EIQ-110 (0.50), EIQ-105 (0.61), EIQ-113 (0.64) and EIQ-106 (0.83) in E2 exhibited positive significant GCA effects. Specific combining ability effects revealed that out of forty- five hybrids, seventeen hybrids in E1 with the magnitude ranged from 1.35 (EIQ-107 x EIQ-110) to 8.00 (EIQ-111 $x$ EIQ-114) and twelve hybrids in E2 with the magnitude ranged from 2.09 (EIQ-105 $\mathrm{x}$ EIQ-114) to 6.06 (EIQ-109 x EIQ-110) exhibited positive significant SCA effects.

The estimates of GCA effects for grain yield per plant revealed that only three inbred lines in E1 with the magnitude of 3.05 (EIQ-106), 8.89 (EIQ-109) and 10.46 (EIQ-112) and only two inbreds in E2 with the magnitude of 6.01 (EIQ-112) and 6.79 (EIQ-109) showed positive significant GCA effects. The estimates of positive significant SCA effects for grain yield per plant were depicted by twenty and seventeen hybrids in E1 and E2 environments respectively. The range in E1 was 4.41 (EIQ-110 x EIQ-113) to $34.59 \mathrm{~g}$ (EIQ-110 x EIQ-112) while, the range in E2 varied from 7.78 (EIQ-110 x EIQ-113) to 27.95 (EIQ-110 x EIQ-112). For Harvest Index three inbred lines in E1 viz., EIQ-112 (0.87), EIQ-109 (0.93) and EIQ-106 (1.13) and four inbred lines in E2 environment viz., EIQ-109 (0.52), EIQ-110 (0.65), EIQ-113 (0.67) and EIQ-106 (0.72) expressed significant GCA effects for harvest index.

The estimates of SCA effects revealed that sixteen hybrids in E1 exhibited positive significant SCA effects with the magnitude varied from 1.69 (EIQ-109 x EIQ-114) to 9.11 (EIQ-105 x EIQ-113) and in E2 also sixteen hybrids exhibited positive significant SCA effects with the magnitude varied from
1.70 (EIQ-109 x EIQ-111) to 9.81 (EIQ-105 $x$ EIQ-113). Over the environment analysis revealed that only three inbred lines viz., EIQ-113 (0.57), EIQ-109 (0.72) and EIQ106 (0.93) expressed significant GCA effects. By the estimates of SCA effects eighteen hybrids with the magnitude ranged from 1.30 (EIQ-105 x EIQ-107) to 9.46 (EIQ-105 x EIQ-113) exhibited positive significant SCA effects.

The GCA effects represent the additive nature of gene action. A good general combiner parent is characterized by its better breeding value when crossed with other parents. Depending upon the character concerned the nature (direction/sign) and per se (mean) performance of the parent is also considered in association with GCA since the former offers reliability and authenticity to GCA as a guide to selection of the parent. On the basis of per se performance and GCA effects good general combiner lines were identified and presented in (Table 1).

On the basis of mean and SCA effects out of total 45 hybrids, five hybrids which exhibited higher mean and significant positive SCA effects for grain yield per plant were EIQ-110 x EIQ-112, EIQ-107 x EIQ-109, EIQ-109 x EIQ-111, EIQ-105 x EIQ-112 and EIQ-105 $x$ EIQ-114 in $E_{1}$ environment while hybrids EIQ-110 x EIQ-112, EIQ-105 x EIQ-112, EIQ-109 x EIQ-111, EIQ-107 x EIQ-109,

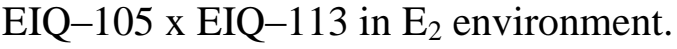

Among these the crosses EIQ-110 x EIQ112, EIQ-107 x EIQ-109, EIQ-109 x EIQ111, EIQ-105 x EIQ-112 were better performer in both the environment, so these lines can be recommended for multi-location trials. By the study, it can be concluded that the crosses having best specific combination for grain yield per plant would obtained either through poor x good, average $\mathrm{x}$ good or poor $\mathrm{x}$ poor parental combinations. 


\section{References}

Aguir, A. M., Carlini-Garcia, L. A., Silva, A. R., Santos, M. F., Garcia, A. A. F. and Desouja, C. L. 2003. Combining ability of inbred lines of maize and stability of their respective single crosses. Scientia Agricola. 60: 83-89.

Alabi, S.O., Obilana, A. B. and Nwasike, C.C. 1987. Gene action and combining ability for quantitative characters in upland cotton. Samaru Agric. Res. 5(12): 59-64.

Alamerew, S., and Warsi, M.Z.K. 2015. Heterosis and Combining Ability of Sub Tropical Maize Inbred Lines. African Crop Science Journal. 23(2):123 - 133.

Annual progress report, AICRP on Maize 2015: Indian Institute of Maize Research, New Delhi.

Bartlett, M. S., 1937. Properties of sufficiency and statistical tests. Proc. Roy. Soc. London A 160 268-282.

Bello, O. B., and Olaoye, G. 2009. Combining ability for maize grain yield and other agronomic characters in a typical southern guinea savanna ecology of Nigeria. African Journal of Biotechnology, 8 (11): 2518-2522.

Chawla, H.S., and V.P. Gupta, 1984. Index India-Agric. Indian Calcutta Agric. 28(4): 261- 265.

Dar, Z. A., Lone, A. A., Alaie, B. A., Ali, G., Gazal A., and Abidi, I. 2015. Estimation Of Combining Ability Involving Quality Protein Maize (Qpm) Inbreds Under Temperate Conditions. The Bioscan, 10(2): 863-867.

Griffing, B., 1956. Concept of general and specific combining ability in relation to diallel crossing systems. Australian Journal of Biological Sciences, 9: 463493.

Haddadi, M. H., Eesmaeilof, M., Choukan, R. and Rameeh, V. 2012. Combining ability analysis of days to silking, plant height, yield components and kernel yield in maize breeding lines. African $J$. Agric. Res. 7(33): 4685-4691.

Izhar, T., and Chakraborty, M. 2013. Combining ability and heterosis for grain yield and its components in maize inbreds over environments (Zea mays L.). Afr. J. Agric. Res. 8(25):3276-3280.

Joshi, V.N., Pandiya NK, Dubey RB 1998. Heterosis and Combining ability for quality and yield in early maturing single cross hybrids of maize. Indian $J$. Genet. Plant Breed. 58: 519-524.

Keskin, B., Yilmaz IH, Turan N. 2005. Yield and quality of forage corn (Zea mays L.) as influenced by cultivar and nitrogen rate. Journal of agronomy. 4(2):138141.

Krupakar, A., Kumar, B. and Marker, S. 2013. Combining ability for yield and quality traits in single cross hybrids of maize (Zea mays L.). The Bioscan. 8(4): 1347-1355.

Kumar, N., Joshi, V. N. and Dagla, M. C. 2013. Estimation of components of genetic variance in maize (Zea mays L.). The Bioscan. 8(2): 503-507.

Lal, J. J., and Kumar, R. S. 2012. Combining Ability and Heterosis for Polygenic Characters in Maize (Zea mays L.). Madras Agric. J., 99(4-6):174-177.

Moneam, A. M. A., Attia, A. N., Emery-El, M. I. and Fayed, E. A. 2009. Combining ability and heterosis for some agronomic traits in crosses of maize. Pakistan Journal of Biological Sciences. 12 (5): 433-8.

Nass, L.L., Lima, M., Vencovsky, R. and Gallo, P.B. 2000. Combining ability of maize inbred lines evaluated in three environments in Brazil. Science and Agriculture 57:129-134.

Poehlman, J. M., 2006. Breeding Field Crops. 5th Edn. The AVI publish. Co. Inc. Westport, Connecticut.

Qi. X., Kimatu, J.N., Li, Z., Jiang, L., Cui, Y 
and Liu, B. 2010. Heterotic analysis using AFLP markers reveals moderate correlation between specific combining ability and genetic distance in maize inbred lines. African Journal of Biotechnology 9:1568-1572.

Singh, D., 1973. Diallel analysis for combining ability over several environments. Indian Journal of Genetics, 33: 469-81.

Singh, D., 1979. Diallel analysis for combining ability over environments. Indian Journal of Genetics, 39: 383-86.

Sprague, G. H., and Tatum, L. A. 1942. General v/s specific combining ability in single crosses in corn. Journal of American Society of Agronomy, 30: 923-932.

USDA-FAS, 2014. United States Department of Agriculture, Foreign Agricultural Service.

Vasal, S.K., Srinivasan G, Beck DL, Cross J, Pandey S, Deloen C 1992. Heterosis and combining ability of CIMMYT's tropical late white maize germplasm. Maydica, 37: 217-223.

Weatherwax, Paul 1955. History and origin of corn. In G.F. Spragne (Ed.). Corn and corn improvement, Academic Press. New York. 1-16.

\section{How to cite this article:}

Avinash Kumar, Amit Dadheech, N. Kiran, Prashant Bisen and Santosh Kumar. 2017. Diallel Analysis of Combining Ability for Yield and Yield Contributing Traits over the Environments in Maize (Zea mays L.). Int.J.Curr.Microbiol.App.Sci. 6(10): 196-208.

doi: https://doi.org/10.20546/ijcmas.2017.610.025 